\section{Arnaud Ferry Michel Rieu}

\section{ADRESSE}

A. Ferry : professeur des universités à l'UFR sciences et techniques des activités physiques et sportives. M. Rieu : professeur de physiologie à la faculté de médecine Cochin-Port-Royal. Laboratoire de physiologie des adaptations, université Paris V, 24, rue du Faubourg-

\title{
Adaptation des systèmes physiologiques à l'activité physique
}

La course de type "endurance " sur tapis roulant motorisé chez le rat, principal modèle animal d'exercice musculaire, induit les mêmes adaptations du transport de l'oxygène et du système musculaire que celles observées chez le sportif humain. Ce modèle animal d'activité physique devrait permettre l'étude des processus cellulaires et moléculaires adaptatifs impliqués dans la plasticité musculaire (régulation de l'expression des gènes de la myosine, des enzymes mitochondriales...). Les résultats de ces études devraient permettre, en outre, de proposer les mesures appropriées pour lutter contre la désadaptation physiologique qui apparaît lors d'une sédentarité forcée (sujet hospitalisé, greffé, spationaute...) ou au cours du vieillissement.

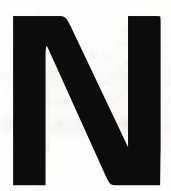
ous focaliserons notre attention sur un type particulier d'activité physique défini par le terme d'endurance et dont le prototype est le marathon $(42,3 \mathrm{~km})$. Les réponses physiologiques du marathonien ont été récemment décrites [1]. Cet exercice musculaire est réalisé à une intensité modérée (la consommation maximale d'oxygène n'est pas atteinte) pendant plus de deux heures. Plusieurs milliers de Français pratiquent, plus ou moins régulièrement, ce type d'effort dans le cadre de la compétition sportive, des loisirs ou encore dans celui de la santé publique [2]. C'est d'ailleurs un adjuvant thérapeutique de plus en plus prisé de certaines maladies métaboliques (principalement le diabète) ou cardiovasculaires [3, 4]. Toutefois, afin d'explorer les mécanismes cellulaires et moléculaires impliqués dans la réponse de l'organisme à l'exercice musculaire, les physiologistes ont dû développer des modèles animaux mimant l'activité physique humaine, principalement du fait de la nature "invasive" de certaines techniques utilisées.

\section{Pertinence \\ des modèles animaux}

Dans la littérature internationale, plusieurs modèles animaux d'exercice musculaire ont été décrits. Que ce soit pour déterminer l'effet isolé ou aigu (induit par une seule séance d'effort) ou l'effet chronique (induit de façon durable par l'entraînement physique) de l'exercice musculaire, ces modèles animaux ont été développés principalement chez le ron- 
geur et plus particulièrement chez le rat. Le plus utilisé est la course sur tapis roulant motorisé. La vitesse de la course de l'animal ainsi que sa durée sont rigoureusement réglées en fonction de l'âge, des capacités physiques des animaux et des objectifs expérimentaux (effort d'endurance modéré, intense, voire même conduisant à l'épuisement physique), de telle sorte que ce modèle induit des effets parfaitement reproductibles. Mais ce modèle animal d'exercice musculaire est-il pertinent : la course sur tapis roulant chez le rat reproduit-elle les effets de la course chez l'humain ? Répondre à cette question nécessite de comparer leurs effets respectifs sur les grands systèmes physiologiques : neuroendocrinien (Tableau I) [5], cardiovasculaire (Tableau II) [6-10], locomoteur (muscles des membres inférieurs) (Tableau III) [8, 9, 11-13] et immunitaire (Tableau IV) [14, 15]. Dans l'état actuel des connaissances, on peut conclure que la course sur tapis roulant chez le rat est un modèle globalement satisfaisant de la course chez l'humain, car l'effet chronique de la course sur tapis roulant chez le rat reproduit les mêmes adaptations que celles observées chez l'humain entraîné à la course d'endurance. Ces dernières conduisent, principalement, d'une part, à l'augmentation du transport maximal de l'oxygène via celle du volume maximal d'éjection systolique [7] et, d'autre part, à l'accroissement des capacités oxydatives des fibres musculaires squelettiques recrutées lors de l'effort $[8,9$, 11]. Cette augmentation du métabolisme oxydatif musculaire résulte du nombre plus élevé de mitochondries et de fibres musculaires squelettiques de type IIa (fibres à contraction lente et au métabolisme principalement oxydatif) et d'une capillarisation musculaire plus importante. La même conclusion peut être réalisée à propos de l'effet isolé (aigu) de la course puisqu'une seule séance d'exercice chez le rat induit les mêmes réponses de la part des systèmes physiologiques que celles observées chez l'humain, en particulier : augmentation des concentrations sanguines des catécholamines et des glucocorticoïdes, et accroissement du transport de l'oxygène et du métabolisme énergétique muscu- laire (Tableaux I, II et III). On peut également noter que l'exercice musculaire n'affecte pas seulement les systèmes physiologiques directement responsables, a priori, de l'activité motrice (neuroendocrinien, cardiovasculaire et musculaire), mais également d'autres systèmes, tel le système immunitaire (Tableau IV). Par exemple, l'exercice isolé mobilise dans le sang certaines sous-populations de cellules $\mathrm{T}\left(\mathrm{CD}^{+}\right)$ou les cellules tueuses naturelles (NK) depuis des compartiments de l'organisme non encore identifiés avec certitude. De plus, cette modulation des effectifs des cellules immunocompétentes induite par l'exercice est également associée à des modifications des fonctions in vitro de ces cellules (réponse proliférative aux mitogènes, production de cytokines, activité cytotoxique...) [14-16]. Les mécanismes à l'origine des modulations immunologiques associées à l'exercice sont vraisemblablement neuroendocriniens (catécholamines, glucocorticoïdes...) $[15,16]$.

En dehors de la course sur tapis roulant, on peut également forcer le rat à nager dans un bassin. Mais ce type de stress - conduisant à des réponses hormonales globalement similaires à celles de la course sur tapis roulant (bien que parfois plus accentuées) peut-il être, également, considéré comme un modèle d'exercice musculaire? En comparant les effets chroniques de la nage et de la course sur tapis roulant, on constate que ces deux modèles animaux induisent des réponses physiologiques qui diffèrent de façon notable. En effet, contrairement à la course sur tapis roulant, la nage induit d'importantes modifications du myocarde : hypertrophie, augmentation du pourcentage de l'isoforme Vl de la myosine dans le ventricule (isoforme portant l'activité ATPasique la plus élevée), et accroissement de la densité mitochondriale et de certaines activités enzymatiques du cycle de Krebs et de la chaîne respiratoire $[6,10]$. On ignore actuellement si de telles modifications pourraient être observées chez l'homme. De plus, la nage chez le rat n'induit aucun changement notable au niveau des muscles des membres inférieurs, contrairement à la course sur tapis roulant [8, 10]. A la lumière de ces résultats divergents et compte tenu du fait que la nage chez le rat est une forme de stress physique dont on peut difficilement faire varier l'intensité (contrairement à la course sur tapis roulant), et qui comporte de fréquentes phases d'apnée, il ne semble pas toujours justifié de l'utiliser comme modèle d'exercice musculaire d'endurance, du moins dans le cadre de l'étude des processus adaptatifs au niveau du système locomoteur.

Par ailleurs, compte tenu du nombre croissant de publications internationales utilisant ce procédé, il faut s'interroger sur la pertinence de l'électrostimulation de basse fréquence $(10 \mathrm{~Hz})$ du muscle squelettique. Ce modèle, appliqué au rat, conduisant vraisemblablement à la contraction de toutes les fibres musculaires de type I (lentes et oxydatives), reproduit-il les mêmes effets que le fonctionnement musculaire in vivo lors de la course? Bien que la forme de cette contraction musculaire ne soit que de nature concentrique (contraction avec raccourcissement du muscle), contrairement à la course sur tapis roulant, il ressort que l'électrostimulation induit globalement les mêmes adaptations musculaires que la course sur tapis roulant $[17,18]$. Toutefois, ce modèle d'hyperactivité musculaire n'affecte probablement que le muscle stimulé et non les autres muscles ni les systèmes neuroendocrinien et cardiovasculaire. De plus, on peut noter que les séances d'activité musculaire induite par stimulation électrique de basse fréquence sont d'une durée très nettement supérieure $(10 \mathrm{~h} / 24 \mathrm{~h})$ à celle imposée dans la course sur tapis roulant $(1-2 \mathrm{~h} / 24 \mathrm{~h})$. Il faut bien distinguer, enfin, ces modèles d'activité motrice soutenue (course sur tapis roulant, nage, électrostimulation de basse fréquence), du modèle d'hypertrophie compensatoire résultant de l'ablation des muscles synergistes, car ce dernier provoque une hypertrophie très marquée du muscle squelettique [19].

\section{Perspectives}

A la lumière de quelques très récentes publications, le modèle animal de la course sur tapis roulant chez le rongeur promet d'être un 


\section{RÉFÉRENCES}

1. Rieu M. La biologie du sportif. $L a$ recherche $1992 ; 245: 878-87$.

2. Rieu M. Santé, sédentarité et activité physique. Santé publique $1990 ; 4$ : 26-35.

3. Galbo $H$, von Linstow $M$, Dela $F$, Kjaer M. Exercise and diabetes. In : Sato $Y$, Poortmans J, Hashimoto I, Oshida Y, eds. Integration of medical and sports sciences. Basel : Karger, 1992 : 227-36.

4. Haskell WL, Leon AS, Caspersen CJ, Wilson PWF. Cardiovascular benefits and assessment of physical activity and physical fitness in adults. Med Sci Sport Exerc 1992 ; 24 (suppl) : S201-20.

5. Pesquies PC, Guezennec CY. Commande hormonale du métabolisme énergétique lors de l'exercice musculaire. In : Rieu M, ed. Bioénergétique de l'exercice musculaire et de l'entraînement physique. Paris : Presses Universitaires de France, $1988: 271-87$.

6. Baldwin KM. Effects of chronic exercise on biochimical and functional properties of the heart. Med Sci Sports Exerc 1985; 17 : 522-8.

7. Scheuer J, Tipton CM. Cardiovascular adaptations to physical training. Annu Rev Physiol 1977 ; 39 : 221-51.

8. Baldwin KM, Cooke DA, Cheadle WG. Time course adaptations in cardiac and skeletal muscle to different running programs. J Appl Physiol 1977 ; 42 : 267-72.

9. Booth FW, Thomason DB. Molecular and cellular adaptation of muscle in response to exercise : perspectives of various models. Physiol Rev $1991 ; 71$ : 54174.

10. Pagani ED, Solaro RJ. Swimming exercise, thyroid state, and the distribution of myosin isoenzymes in rat heart. $A m J$ Physiol 1983 ; 245 : H713-H20.

11. Holloszy JO, Coyle EF. Adaptations of skeletal muscle to endurance exercise and their metabolic consequences. $J A p p l$ Physiol $1984 ; 56: 831-8$.

12. Fitzimons DP, Diffee GM, Herrick RE, Baldwin KM. Effects of endurance exercise on isomyosin patterns in fast- and slowtwitch skeletal muscles. J Appl Physiol 1990 ;
Tableau I

EFFET DE LA COURSE D'ENDURANCE SUR LE SYSTĖME NEUROENDOCRINIEN

\begin{tabular}{lcc|}
\hline & Rat & Homme \\
\hline $\begin{array}{l}\text { Effet de l'exercice chronique } \\
\text { CRF-ACTH-glucocorticoïdes }\end{array}$ & - & idem \\
LH, FSH-androgènes sexuels & $=,+,-$ & idem \\
TSH-hormones thyroïdiennes & & idem \\
\hline $\begin{array}{l}\text { Effet de l'exercice isolé } \\
\text { Catécholamines }\end{array}$ & + & idem \\
Insuline & + & idem \\
Hormone de croissance & + & - \\
CRF-ACTH-glucocorticoïdes & - & idem \\
LH, FSH-androgènes sexuels & & \\
TSH-hormones thyroïdiennes ? & $=,+$ & \\
\hline
\end{tabular}

$=$ : pas de variation ; + : stimulation ; - inhibition ; ? non determiné. D'après $[1,5,9]$

\begin{tabular}{|c|c|c|}
\hline \multicolumn{3}{|c|}{$\begin{array}{l}\text { EFFET DE LA COURSE D'ENDURANCE } \\
\text { SUR LE SYSTEME CARDIOVASCULAIRE }\end{array}$} \\
\hline & Rat & Homme \\
\hline $\begin{array}{l}\text { Effet de l'exercice musculaire } \\
\text { Fréquence cardiaque } \\
\text { Volume éjection systolique } \\
\mathrm{VO}_{2} \text { max (à la bouche) } \\
\mathrm{Masse} \text { cardiaque } \\
\text { Myosine }{ }^{1} \\
\text { Enzymes oxydatives (V }{ }_{\text {max }} \text { ) }\end{array}$ & $\begin{array}{l}+ \\
+ \\
+ \\
+(\text { femelle }) \\
= \\
=\end{array}$ & $\begin{array}{c}\text { idem } \\
\text { idem } \\
\text { idem } \\
\text { idem } \\
? \\
?\end{array}$ \\
\hline $\begin{array}{l}\text { Effet de l'exercice isolé } \\
\text { Fréquence cardiaque } \\
\text { Volume d'éjection systolique } \\
\mathrm{VO}_{2} \text { (à la bouche) }\end{array}$ & $\begin{array}{l}+ \\
+ \\
+\end{array}$ & $\begin{array}{l}\text { idem } \\
\text { idem } \\
\text { idem }\end{array}$ \\
\hline
\end{tabular}

1 : isoformes du ventricule (V1-V3).

D'après [6-10].

augmentation ; - : diminution ; = : sans changement ; ? non déterminé.

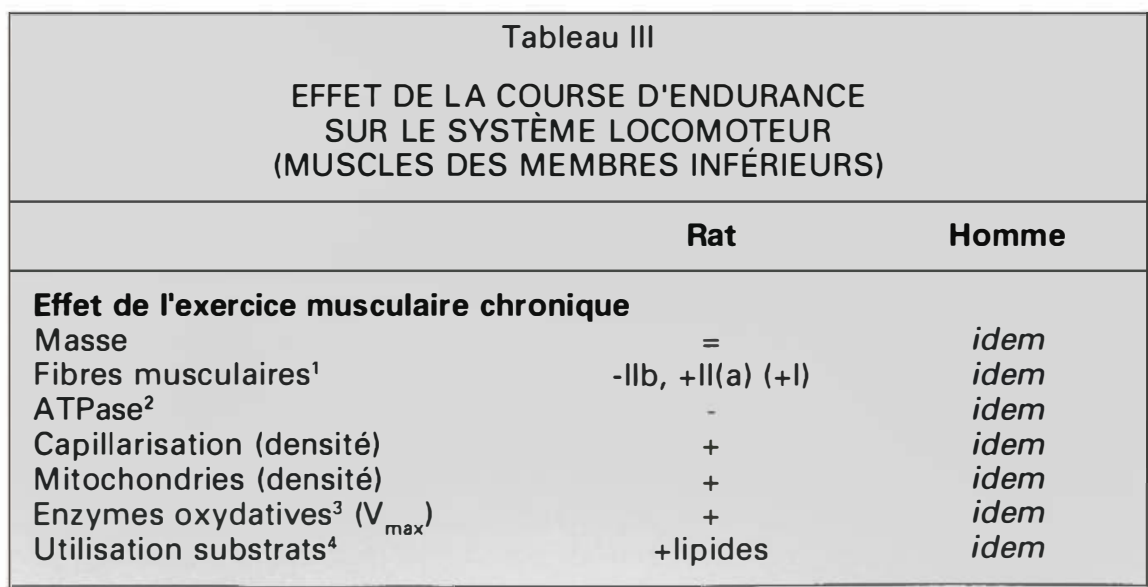

: pourcentage de fibres I/b (rapides et glycolytiques), I (lentes et oxydatives) et lla (intermédiaire), selon la classification histo-colorimétrique (myosine ATPase acido- ou alcalino- résistante).

2 : activité ATPase de la myosine portée par les chaînes lourdes de la myosine.

3 : enzymes du cycle de Krebs et de la chaîne respiratoire.

$"$ : davantage d'acides gras sont oxydés au détriment du pyruvate.

: augmentation ; - : diminution ; =: sans changement. D'après [8, 9, 11-13]. 
Tableau IV

EFFET DE LA COURSE D'ENDURANCE SUR LE SYSTĖME IMMUNITAIRE

\begin{tabular}{|c|c|c|}
\hline & Rat & Homme \\
\hline \multicolumn{3}{|c|}{ Effet de l'exercice musculaire chronique } \\
\hline Circulantes ${ }^{1}$ & $=$ & idem \\
\hline Thymus ${ }^{2}$ & $=,-$ & $?$ \\
\hline \multicolumn{3}{|c|}{ Fonctions in vitro des cellules immunitaires ${ }^{3}$} \\
\hline Circulantes ${ }^{1}$ & $?$ & $=$ \\
\hline Rate $^{4}$ & + & $\bar{?}$ \\
\hline \multicolumn{3}{|c|}{$\begin{array}{l}\text { Effet de l'exercice isolé } \\
\text { Effectifs des cellules immunitaires circulantes }{ }^{1}\end{array}$} \\
\hline & + & idem \\
\hline \multicolumn{3}{|c|}{ Fonctions in vitro des cellules immunitaires } \\
\hline Circulantes ${ }^{2}$ & ? & - \\
\hline Rate $^{4}$ &,$+=,-$ & $?$ \\
\hline
\end{tabular}

: principalement lymphocytes $T$ et cellules natural killer.

: organe lymphoïde primaire où se fait la maturation/différenciation des lymphocytes $T$.

${ }^{3}$ : activité cytotoxique des cellules $T$ ou des cellules natural killer, réponse proliférative des lymphocytes $T$...

‘: I'un des principaux organes lymphoïdes secondaires.

: augmentation ; -: diminution ; =: sans changement ; ? : non-déterminé. D'après : [14-16].

\section{Tableau V}

EFFET DE LA COURSE D'ENDURANCE SUR LE SYSTĖME RESPIRATOIRE (DIAPHRAGME)

\begin{tabular}{|c|c|c|}
\hline & Rat & Homme \\
\hline \multicolumn{3}{|c|}{ Effet de l'exercice musculaire chronique } \\
\hline Fibres musculaires & (\%) & \\
\hline crural & $=$ & $?$ \\
\hline costal & $+11 b-11 a$ & $?$ \\
\hline Capillarité (costal) & $=,+11 b$ & $?$ \\
\hline Enzymes oxydatives ${ }^{1}\left(V_{\max }\right)$ & + & $?$ \\
\hline crural & $=$ & $?$ \\
\hline costal & + & $?$ \\
\hline
\end{tabular}

enzymes du cycle de Krebs et de la chaîne respiratoire.

augmentation ; - : diminution ; = : sans changement ; ? : non-déterminé. D'après [29, 31, 32].

modèle physiologique de premier ordre pour l'étude des processus cellulaires et moléculaires adaptatifs impliqués dans la plasticité musculaire. A titre d'exemple, il a été montré que l'effet chronique de la course sur tapis roulant chez le rat induit la synthèse de la cytochrome oxydase (enzyme de la chaîne respiratoire) dans le muscle squelettique, sans modification de la quantité des ARN messagers de ses sous-unités [20]. De tels changements $m / s n^{\circ} 8 / 9$ vol. 10, août-septembre 94 régulations induites par l'exercice musculaire chronique - qui sont spécifiques des muscles (lent/rapide, locomoteur/diaphragme) - conduisent à des modifications de la quantité des différentes isoformes des chaînes lourdes (MHC, myosin heavy chains) et légères (MLC, myosin light chains) de la myosine dans les muscles locomoteurs : certaines isoformes caractéristiques des fibres musculaires rapides de type IIb (MHC IIb, LCls, LC2f et LC3f) sont remplacées par des isoformes caractéristiques des fibres musculaires lentes de type I et intermédiaires de type IIa (MHCIIx, MHC IIa, et LC2s). Ces dernières expliquent les transitions, du même ordre, des isoformes de la myosine native (diminution de l'isoforme de myosine FM3 et augmentation de l'isoforme IM) $[12,13]$. Ces transitions d'isoformes des chaînes lourdes de myosine sont évidemment suivies de modifications du mode de contraction de la fibre musculaire (lent, intermédiaire ou rapide) dans la mesure où ce sont ces chaînes lourdes qui portent l'activité ATPasique. On constate également, chez le rat entraîné en endurance, une augmentation quantitative du principal transporteur du glucose du muscle strié squelettique, GLUT-4 [21]. De plus, une seule séance d'exercice conduit à la translocation du GLUT-4 du compartiment microsomal à la membrane plasmique [22]. L'étape limitante de l'utilisation du glucose comme substrat énergétique paraissant être son transport, on comprend l'intérêt que représente l'entraînement physique pour le sujet diabétique. Il reste malgré tout à déterminer les facteurs contrôlant l'expression de cette protéine.

Mais ce modèle d'exercice musculaire (course sur tapis roulant) peut également avoir quelques effets néfastes. Il peut activer la mort par apoptose des thymocytes [23] ou, encore, induire la mort par nécrose de certaines fibres musculaires recrutées lors de l'effort [24]. Dans le cas de la nécrose des fibres musculaires - 


\section{RÉFÉRENCES}

13. Guezennec CY Gilson E Serrurier B. Comparative effects of hindlimb suspension and exercise on skeletal muscle myosin isoenzymes in rats. Eur J Appl Physiol 1990 ; $60: 430-5$.

14. Shephard RJ, Verde TJ, Thoams SG, Shek P. Physical activity and the immune system. Can J Spt Sci 1991 ; 16 : 163-85.

15. Ferry A. Immunomodulations liées à l'exercice musculaire : les modulations des effectifs des cellules immunocompétentes. Sci Motr 1991 ; 13 : 48-56.

16. Ferry A. Immunomodulations liées à l'exercice musculaire : les modulations des activités des cellules immunocompétentes. Sci Motr $1991 ; 14: 46-53$.

17. Pette D, Staron R. Cellular and molecular diversities of mammalian skeletal muscle fibers. Rev Physiol Biochem Pharmacol $1990 ; 116: 2-47$.

18. Bar A, Simoneau JA, Pette D. Altered expression of myosin light-chain isoforms in chronically stimulated fast-twitch muscle of the rat. Eur J Biochem $1989 ; 178: 591-4$.

19. Tsika RW, Baldwin KM. Time course adaptations in rat skeletal muscle isomyosins during compensatory growth and regression. I Appl Physiol 1987; 63 : 2111-21.

20. Town GP, Essig DA. Cytochrome oxydase in muscle of endurance-trained rats : subunit mRNA contents and heme synthesis. $J A p p l$ Physiol 1993; $74: 192-6$.

21. Slentz CA, Gulve EA, Rodnick KJ, Henriksen EJ, Youn JH, Holloszy JO. Glucose transporters and maximal transport are increased in endurancetrained rat soleus. I Appl Physiol $1992 ; 73$ : 486-92.

22. Goodyear LJ, Hirshman MF, Horton ES. Exercise-induced translocation of skeletal muscle glucose transporters. Am J Physiol 1991 ; 261 : E795-9.

23. Concordet JP, Ferry A. Physiological programmed cell death in thymocytes is induced by physical stress (exercise). Am J Physiol 1993 ; 265 : C626-9.

24. Olgivie RW, Armstrong RB, Baird KE, Bottoms CL. Lesions in the rat soleus exercise. Am J Anat 1988 ; 182 : 335-46.

25. Darr KC, Schultz E. Exercise-induced satellite cell activation in growing and mature skeletal muscle. J ApplPhysiol 1987 ; $63: 1816-21$.

26. McCormick KM, Thomas DP. Exerciseinduced satellite cell activation in senescent soleus muscle. J Appl Physiol 1992 ; 72 : 88893.

27. Booth FW, Linderman JK, Kirby CR. Molecular mechanisms of muscle disuse atrophy (and strategies of prevention). In : Sato Y, Poortmans J, Hashimoto I, Oshida Y, eds. Integration of medical and sports sciences. Basel : Karger, 1992 : 142-9.

28. Stebbins CL, Shultz E, Smith RT, Smith EL. Effects of chronic exercise during ageing and muscle and endplate morphology in rats. $J$ Appl Physiol $1985 ; 58$ : 45-51.

29. Powers SK, Lawler J, Criswell D, Lieu FK, Martin D. Aging and respiratory muscle metabolic plasticity : effects of endurance training. $\int$ Appl Physiol $1992 ; 72$ : 1068-72.

30. Advani SV, Geenen D, Malhotra A, Factor SM, Scheuer J. Swimming causes mysosin adaptations in the rat cardiac isograft. Circ Res $1990 ; 67: 780-3$.

31. Suguira T, Morimoto A, Murakami N. Effects of endurance training on myosin heavy-chain isoforms and enzyme activity in the rat diaphragm. Pflugers Arch $1992 ; 421$ : $77-81$.

32. Tamaki N. Effect of endurance training on muscle fiber type composition and capillary supply in rat diaphragm. Eur J Appl Physiol 1987 ; 56 : 127-31.

33. Baracos E. Exercise inhibits progressive growth of the Morris hepatoma 7777 in male and female rats. Can I Physiol Pharmacol $1988 ; 67: 864-70$.

34. Ferry A, Le Page C, Rieu M. Sex as determining factor in the effect of exercise on in vivo autoimmune response, adjuvant arthritis. J Appl Physiol 1994 ; 76 : 1172-5.

35. Le Page C, Ferry A, Rieu M. Effect of muscular exercise on chronic releasing experimental autoimmune encepha. lomyelitis, as in vivo marker of immune d'autant plus marquée que la course est intense (vitesse de course élevée) et que l'effort comporte une composante excentrique importante (contraction musculaire lors de l'étirement du muscle) -, elle est suivie par la régénération des tissus musculaires lésés. Il a été démontré que la libération de protéines musculaires (créatine kinase, myosine...) dans le sang - due à la perte d'intégrité au niveau cellulaire et au niveau de l'ultrastructure de certaines fibres musculaires de type lent [23] - est associée à l'activation des cellules satellites [25, 26], cellules mononucléées situées à la périphérie des fibres musculaires. La prolifération de ces cellules satellites, puis leur fusion, entre elles ou avec les fibres musculaires existantes, participent alors à la régénération du muscle. L'étude de ce cycle de dégénérescence/régénération musculaire induit par l'exercice isolé, en particulier celle des facteurs mitogéniques et de différenciation impliqués dans ce cycle, devrait contribuer à la compréhension de certaines myopathies impliquant ces mêmes phénomènes.

En dehors de ces illustrations d'approches fondamentales concernant en premier lieu la plasticité musculaire, l'utilisation de ce modèle d'exercice musculaire chez le rongeur permet de vérifier dans quelle mesure l'entraînement physique régulier chez le sujet vieillissant, convalescent à la suite d'une hospitalisation prolongée, greffé (cardiaque, rénal...), voire même chez le spationaute, est une mesure appropriée pour lutter contre la désadaptation physiologique qui apparaît chez ces sujets. Celle-ci est caractérisée par une atrophie musculaire, induite par l'altération des différentes fonctions physiologiques, par la diminution de la densité osseuse et de la consommation maximale d'oxygène, et pose de graves problèmes de santé publique, compte tenu du très important handicap physique de tels sujets. Un nombre grandissant d'études expérimentales indiquent que l'exercice musculaire chronique régulier diminue les effets délétères de ces périodes de réduc. tion marquée de l'activité physique 
chez les populations animales correspondantes [27-30]. Par ailleurs, à la lumière de certains travaux récents, il semble que le diaphragme - principal muscle du système respiratoire et seul muscle strié vital - s'adapte de façon étonnante à l'exercice musculaire chronique puisque l'une de ses deux régions (costale) adopte une composition cellulaire plus riche en fibres rapides de type IIb (contrairement aux muscles locomoteurs) (Tableau V) $[29,31,32]$. Ces résultats concernant le diaphragme. S'ils sont transposables à l'homme, peuvent-ils être à l'origine d'une amélioration de la fonction respiratoire chez des patients présentant des déficits dans ce domaine ? Enfin, indépendamment d'une amélioration de l'hygiène de vie, l'entraînement physique régulier chez le rongeur est associé à un accroissement de l'immunité antitumorale [33] ainsi qu'à une diminution du développement de certaines maladies auto-immunes expérimentales telles que les modèles animaux de l'arthrite rhumatoïde et de la sclérose en plaques $[34,35]$.
En conclusion, ce modèle animal d'exercice musculaire chez le rongeur - bien que peu étudié en France, contrairement aux autres nations de niveau socio-économique équivalent au nôtre - représente un modèle pertinent de l'activité physique humaine. Il est aussi prometteur car il permet d'explorer les mécanismes responsables des adaptations cellulaires et moléculaires induites par les modifications du milieu intérieur, associées à l'exercice musculaire. Enfin, il peut permettre de déterminer dans quelle mesure l'entrainement physique est un instrument de réadaptation et de prévention de certains désordres provoqués par la sédentarité

\section{Remerciements}

Les auteurs remercient l'Association française contre les myopathies (AFM) de les avoir invités à réaliser cette synthèse qui a été, en partie, présentée au Colloque national sur les maladies neuromusculaires (juin 1993, Strasbourg).

\section{Summary}

Endurance training and physiological adaptation

The running of rats on a motordriven treadmill is the major animal model of endurance muscular exercise. It induces the same adaptations of oxygen transport and muscular system as those observed in athletes. This model should help to determine the molecular and cellular processes involved in muscular plasticity (regulation of the expression of myosin and mitochondrial enzyme genes...). Moreover, it could be helpful in designing protocols to fight consequences of involuntary sedentariness (chronic bed rest, transplantation, space flights...) or aging.

\section{TIRÉS A PART}

A. Ferry 